\title{
Percutaneous surgery for treatment of epidural spinal cord compression and spinal instability: technical note
}

\author{
Claudio E. Tatsui, MD, ${ }^{1}$ Telmo A. B. Belsuzarri, MD, ${ }^{2}$ Marilou Oro, MBA, FNP-BC, ${ }^{1}$ \\ Laurence D. Rhines, MD, ${ }^{1}$ Jing Li, MD, ${ }^{3}$ Amol J. Ghia, MD, ${ }^{3}$ Behrang Amini, MD, PhD, ${ }^{4}$ \\ Heron Espinoza, RT(R)(MR), ARRT, ${ }^{4}$ Paul D. Brown, MD, ${ }^{3}$ and Ganesh Rao, MD ${ }^{1}$
}

Departments of ${ }^{1}$ Neurosurgery, ${ }^{3}$ Radiation Oncology, and ${ }^{4}$ Diagnostic Radiology, The University of Texas MD Anderson Cancer Center, Houston, Texas; and 'Department of Neurosurgery, Catholic University of Campinas, São Paulo, Brazil

\begin{abstract}
OBJECTIVE An emerging paradigm for treating patients with epidural spinal cord compression (ESCC) caused by metastatic tumors is surgical decompression and stabilization, followed by stereotactic radiosurgery. In the setting of rapid progressive disease, interruption or delay in return to systemic treatment can lead to a negative impact in overall survival. To overcome this limitation, the authors introduce the use of spinal laser interstitial thermotherapy (sLITT) in association with percutaneous spinal stabilization to facilitate a rapid return to oncological treatment.
\end{abstract}

METHODS The authors retrospectively reviewed a consecutive series of patients with ESCC and spinal instability who were considered to be poor surgical candidates and instead were treated with SLITT and percutaneous spinal stabilization. Demographic data, Spine Instability Neoplastic Scale score, degree of epidural compression before and after the procedure, length of hospital stay, and time to return to oncological treatment were analyzed.

RESULTS Eight patients were treated with thermal ablation and percutaneous spinal stabilization. The primary tumors included melanoma $(n=3)$, lung $(n=3)$, thyroid $(n=1)$, and renal cell carcinoma $(n=1)$. The median Karnofsky Performance Scale score before and after the procedure was 60 , and the median hospital stay was 5 days (range 3-18 days). The median Spine Instability Neoplastic Scale score was 13 (range 12-16). The mean modified postoperative ESCC score $(2.75 \pm 0.37)$ was significantly lower than the preoperative score $(4.5 \pm 0.27)$ (Mann-Whitney test, $p=0.0044)$. The median time to return to oncological treatment was 5 days (range 3-10 days).

CONCLUSIONS The authors present the first cohort of SLITT associated with a percutaneous spinal stabilization for the treatment of ESCC and spinal instability. This minimally invasive technique can allow a faster recovery without prejudice of adjuvant systemic treatment, with adequate local control and spinal stabilization.

http://thejns.org/doi/abs/10.3171/2016.8.FOCUS16175

KEY WORDS spinal laser interstitial thermotherapy; spinal metastasis; percutaneous spinal stabilization

$\mathrm{O}$ VER the past several years, advances in cancer treatment have improved the overall survival of patients suffering from various malignancies. Concurrently, the incidence of vertebral column involvement has increased. It is estimated that up to $20 \%$ of cancer patients will develop a spinal metastasis, and about half of them will develop spinal cord compression. ${ }^{13}$ In such cases, the urgency of systemic therapy needs to be weighed against the morbidity of local treatment to optimize patient's management.

We recently reported our initial experience with spinal laser interstitial thermotherapy (sLITT) ${ }^{22}$ as an alternative to limited surgical decompression and stabilization (separation surgery) prior to spinal stereotactic radiosurgery (SRS). Our initial study consisted of 11 patients with epi- dural spinal cord compression (ESCC), without neurological deficits. The median hospital stay was 2 days, and most patients underwent spinal SRS within 3 days of the procedure. We reported improvement in the degree of epidural compression in 9 of 11 patients and one treatment failure, which was managed with circumferential decompression and spinal stabilization. A secondary analysis of one patient treated with sLITT 60 days prior to SRS demonstrated an improvement of almost 2 Gy in the minimum dose prescribed to cover the gross target volume of a metastatic pheochromocytoma affecting T11-12. In this case, we obtained long-lasting local control (currently 18 months) and avoided a thoracolumbar stabilization. ${ }^{9}$

Here, we introduce the concept of percutaneous sepa-

ABBREVIATIONS $\mathrm{cEBRT}=$ conventional external-beam radiation therapy; $\mathrm{ESCC}=$ epidural spinal cord compression; iMRI = intraoperative MRI; $\mathrm{KPS}=\mathrm{Karnofsky} \mathrm{Perfor-}$ mance Scale; SINS = Spine Instability Neoplastic Scale; SLITT = spinal laser interstitial thermotherapy; SRS = stereotactic radiosurgery.

SUBMITTED April 21, 2016. ACCEPTED August 2, 2016.

INCLUDE WHEN CITING DOI: 10.3171/2016.8.FOCUS16175. 


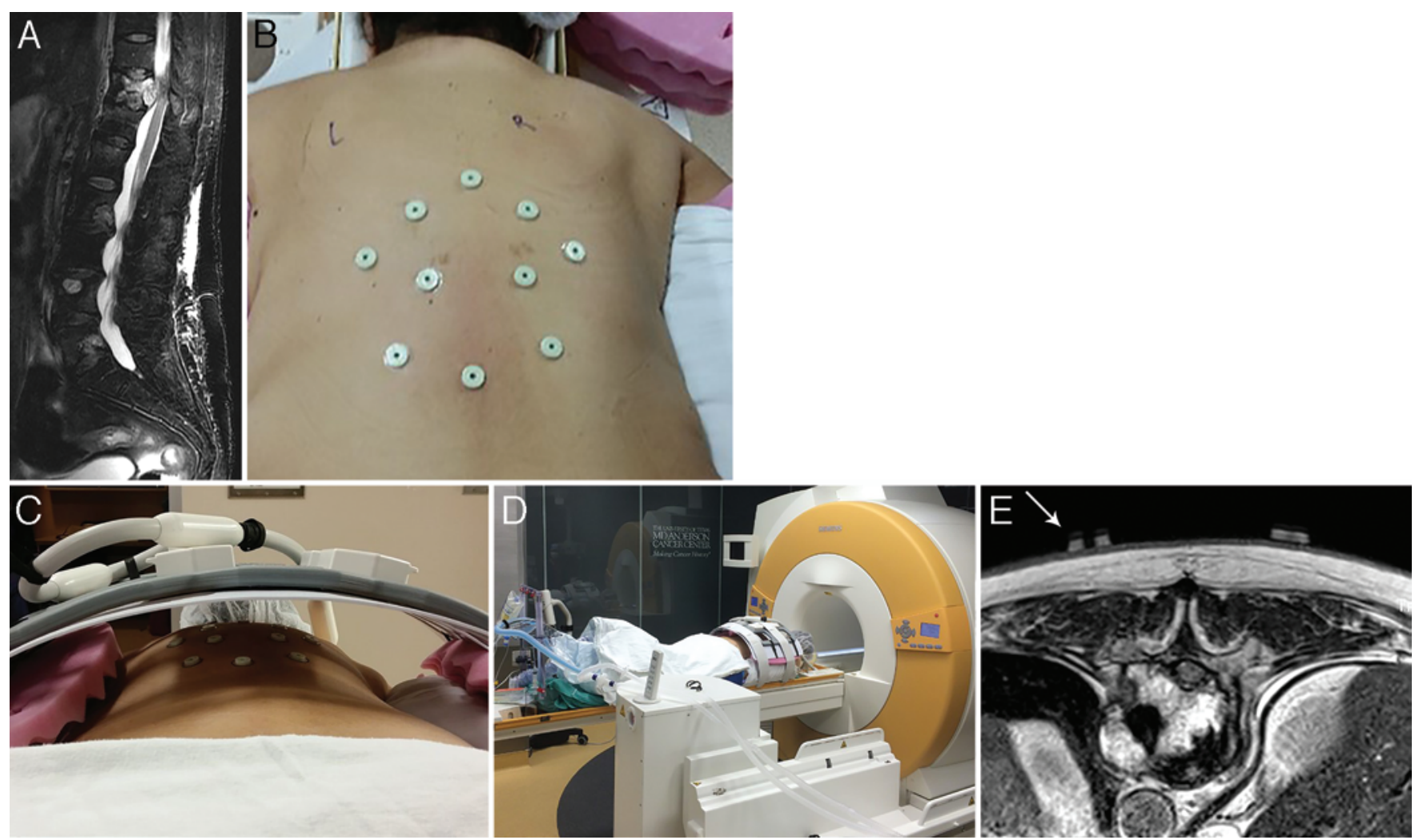

FIG. 1. Case 6. Preparation and acquisition of intraoperative images for MRI-based image guidance. A: Preoperative sagittal T2weighted image of the lumbar spine, demonstrating a pathological fracture of T-11 with complete obliteration of the CSF around the spinal cord from a patient with metastatic follicular carcinoma of the thyroid. B: The patient is positioned prone on the MRI transfer table, and standard fiducial markers are placed overlying the region of interest. C: The MRI coil is taped over a plastic cradle to prevent contact with the skin, avoiding distortion of the position of the fiducials during manipulation of the patient in and out of the MRI scanner. D: The patient is transported in the operative position for acquisition of T2-weighted MRI sequences of the region of interest E: Axial T2-weighted image of the T-11 tumor. Note the easy recognition of the fiducial marker (arrow). These images are exported to a standard image guidance system for surface landmark matching registration.

ration surgery by combining the application of sLITT to minimally invasive percutaneous stabilization with and without cement augmentation of the pedicle screws. We report a series of 8 patients and describe the modifications in the intraoperative workflow, and the short-term analysis of the local control, hospital stay, and time to initiate adjuvant therapy.

\section{Methods}

\section{Patient Population and Evaluation}

This retrospective study was performed under the auspices of the MD Anderson Cancer Center institutional review board. All patients had documented spinal metastasis from histologies considered to be radioresistant, or had previously undergone conventional external-beam radiation therapy (cEBRT) and/or SRS that had failed. All patients were considered to have spinal instability based on their Spine Instability Neoplastic Scale (SINS) score as described by Fisher et al. ${ }^{4}$ The degree of spinal cord compression before and after treatment was scored according to the epidural spinal cord compression scale described by Bilsky et al. ${ }^{2}$ Motor function was evaluated according to the Frankel classification. ${ }^{5}$ All cases were discussed by a multidisciplinary tumor board comprising neurosurgery, radiation oncology, and radiology services. All patients in this study were considered to be poor surgical candidates requiring epidural decompression and spinal stabilization. The interval to initiate adjuvant treatment, hospital stay, and surgical morbidity were recorded. Patients with acute neurological deficits or contraindications for MRI were excluded and referred for surgery.

\section{Image Guidance for Laser Catheter Placement}

The procedure is performed inside the iMRI (Brainlab Inc.) under general anesthesia. The patient is positioned prone, with the arms parallel to the body on the iMRI transfer table. A fluoroscope (Siemens) is positioned inside the iMRI room, at a safe distance to obtain anteroposterior and lateral fluoroscopic images. Fiducial markers (IZI Medical Products) are randomly placed in the dorsal region overlying the area of interest and marked with a surgical pen. A plastic cradle is used to support a Siemens body matrix coil, avoiding contact with the skin and the fiducial markers. The patient is transferred to the iMRI unit and T2-weighted images of the region of interest are obtained, uploaded to the navigation software (Brainlab Inc.), and prepared for surface-matching registration (Fig. 1). The patient is removed from the iMRI unit and placed at 

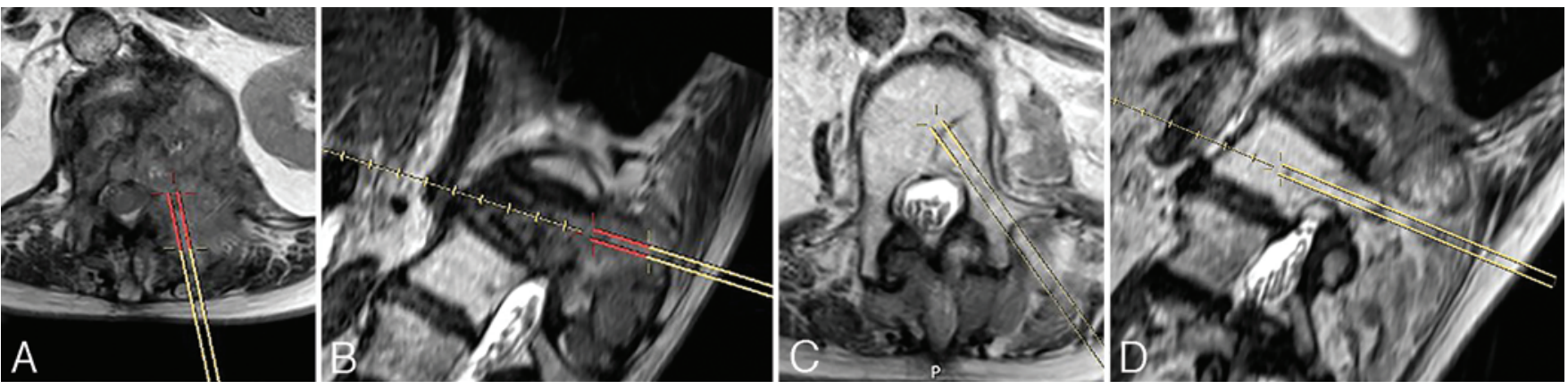

FIG. 2. Case 4. Image guidance based on MR images for laser fiber and pedicle screw placement. A and B: Axial and sagittal inline screenshots demonstrating the placement of the laser fiber. The yellow portion of the virtual needle represents the actual location of the Jamshidi needle. The red portion is an offset, which allows visualization of the trajectory to the final target. At this stage, anteroposterior fluoroscopy is performed, and the position of the needle is compared with the predicted location (image not shown). If the surgeon agrees with the accuracy, the needle is advanced into the final target. C and $\mathbf{D}$ : Axial and sagittal inline views of the image guidance during placement of the percutaneous pedicle screws in the level adjacent to the pathological fracture.

a safe distance away from the high-power magnetic field. A reference array is secured to the patient, and the fiducial markers are registered. Accuracy is verified by applying the navigation tool to the fiducials or at midline using the spinous processes as landmarks. An offset of $60-70 \mathrm{~mm}$ is added to the image guidance, and the inline axial view is used to guide the best trajectory to reach the epidural tumor. A navigated Jamshidi needle (DePuy-Synthes) registered to the navigation system is advanced until it reaches the lamina of the target vertebra. Fluoroscopic images in the anteroposterior projection are obtained and compared with the navigation screen to verify the accuracy using the corresponding pedicle as landmark. The offset is removed, and the Jamshidi is advanced to the final target (Fig. 2). A $\mathrm{K}$-wire is introduced through the Jamshidi needle, which is exchanged for a plastic access cannula. In our experience, each laser fiber can achieve a $10-\mathrm{mm}$ sphere of thermal damage; therefore, multiple needles are positioned in tandem to treat larger tumors (Fig. 3). Finally, we apply sterile towels over the field and cover the cannulas with sterile plastic bags for protection and rapid access. Two Siemens body matrix coils are applied, and the patient is

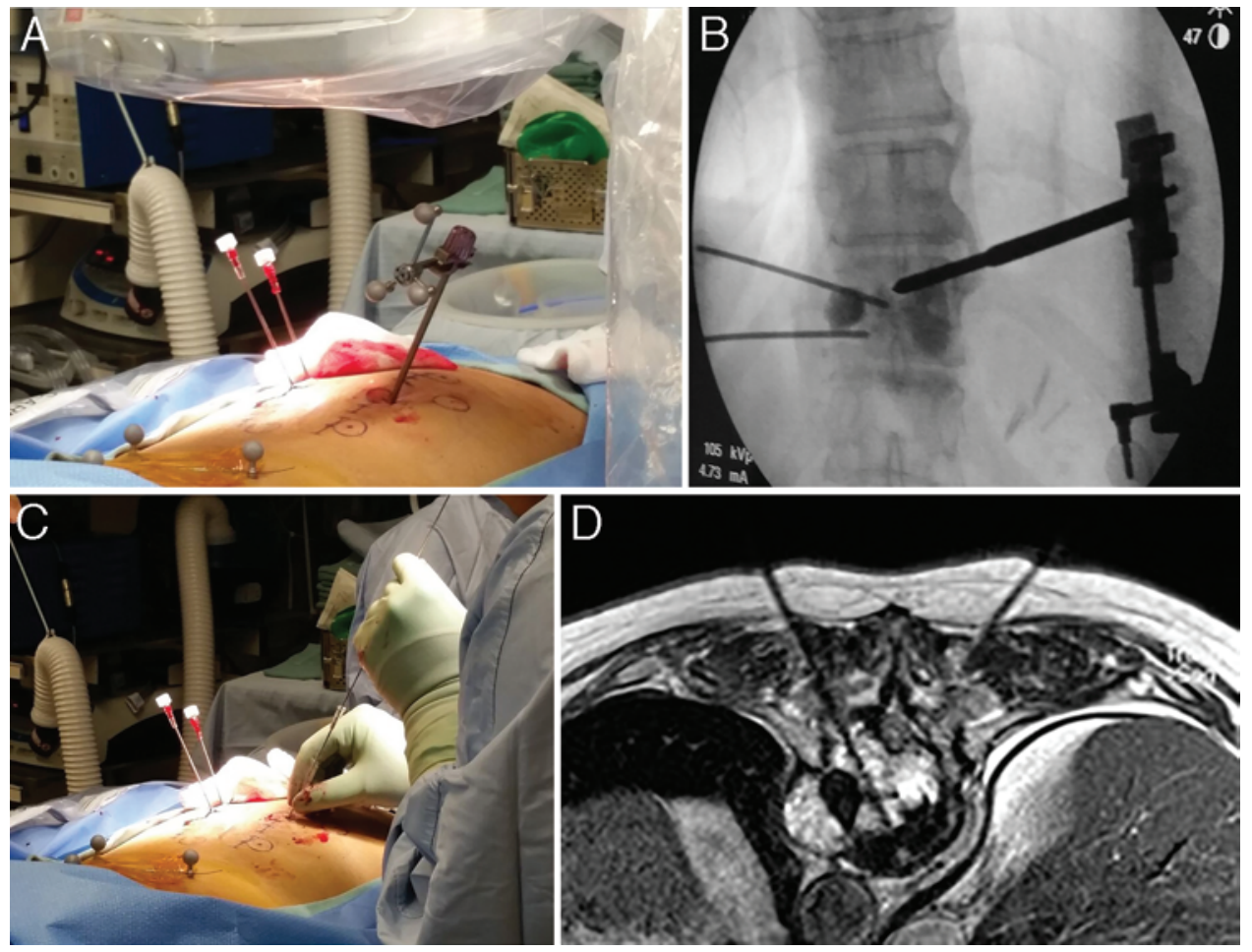

FIG. 3. Placement of the laser fiber. A: The navigated Jamshidi needle is docked in the bone (lamina or pedicle) and anteroposterior fluoroscopy is used to verify accuracy prior to its advancement into the final position. B: Anteroposterior fluoroscopy of a final position demonstrating the needle reached midline. C: The Jamshidi needle is exchanged for a plastic access cannula, and an MRI-compatible titanium needle is used to maintain its position until the placement of the laser fiber. D: Axial image of the exact axial plane of each fiber is stored, and the MRI thermography is overlaid on the same plane during the laser ablation. 


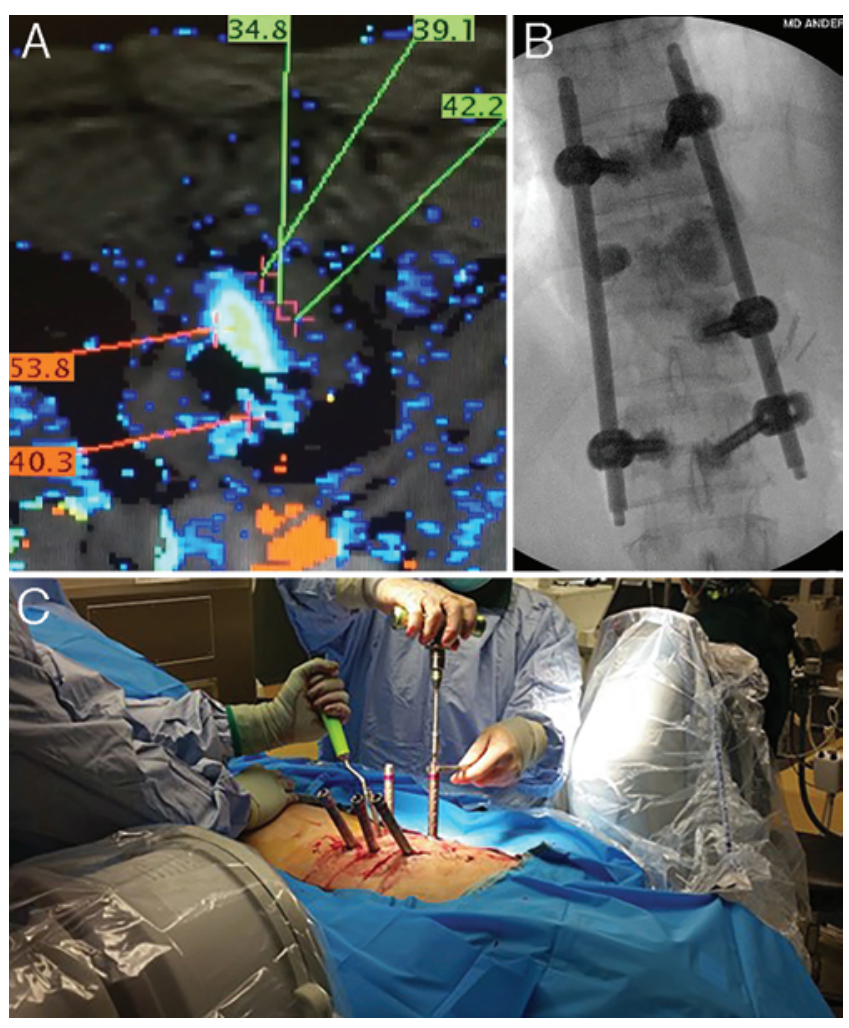

FIG. 4. Intraoperative footage of the percutaneous separation surgery. A: Intraoperative footage of the MRI thermography. The green boxes indicates temperature on the epidural space and the red boxes the temperature near the fiber. B: Anteroposterior fluoroscopic image of the final construct demonstrating adequate positioning of the hardware. C: Intraoperative picture of the placement of the percutaneous instrumentation inside the iMRI room.

transferred to the iMRI unit for localization of the exact axial plane of each access cannula.

\section{Thermal Ablation}

The technique has been previously described. ${ }^{22}$ In brief, heating monitoring is based on MRI gradient-echo acquisition, due to the temperature sensitivity of the proton resonance frequency. This difference in phases estimates the temperature of the tissue on a pixel basis. We use a 38msec TR and $20^{\circ}$ flip angle. The acquisition matrix is 256 $\times 128$ over fields of view of 24-32 cm, with single $3-\mathrm{mm}$ slices acquired every 5-6 seconds. The interface between the dura and the tumor is selected to a temperature limit of $48^{\circ} \mathrm{C}$ to $50^{\circ} \mathrm{C}$ (Fig. $4 \mathrm{~A}$ ). Any movement may deteriorate the thermal map. Therefore, we perform the laser ablation under ventilatory arrest and close monitoring by the anesthesiologist. We limit each cycle of laser activation to no more than 120 seconds, usually repeated 3 times per fiber. Between activations, ventilation is resumed and the $\mathrm{SPO}_{2}$ is returned to $100 \%$.

\section{Estimation of Thermal Damage}

Upon completion of the procedure, we obtain T1weighted images pre- and postcontrast under respiratory arrest. Both images are fused, and the nonenhancing component is subtracted from the image, emphasizing the en-

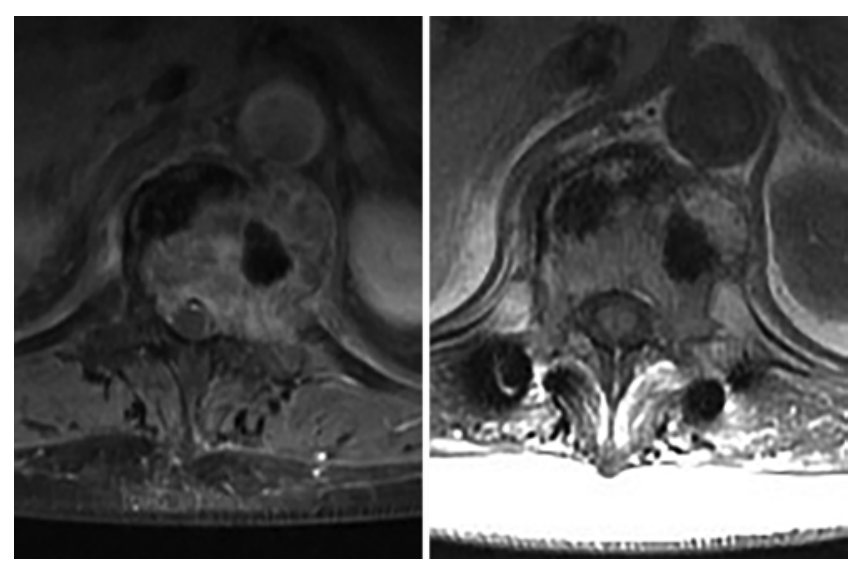

FIG. 5. Imaging follow-up. Left: Preoperative axial T1-weighted sequence with contrast, demonstrating complete obliteration of the CSF around the spinal cord. Right: Axial T1-weighted sequence with contrast obtained 2 months after the operation. The patient started systemic treatment 6 days after the surgical procedure. Note complete resolution of the epidural compression.

hancing tissue. Acute coagulative necrosis is well demonstrated by this technique, and an immediate assessment of the effectiveness of the procedure can be inferred.

\section{Percutaneous Stabilization and Follow-Up}

The patient is removed from the iMRI unit and positioned at a safe distance from the high magnetic field. The reference array is reapplied to the patient, and the outline of the fiducial markers can be used to reregister the parameters of navigation. Alternatively, the percutaneous instrumentation can be inserted using standard fluoroscopic technique. ${ }^{10}$ In cases of severe osteoporosis, we perform cement augmentation of the screws ${ }^{15}$ to increase the purchase in bone, aiming to achieve a more durable stabilization (Fig. 4B and C). Follow-up imaging is performed generally 6-12 weeks after sLITT (Fig. 5).

\section{Statistical Analysis}

Statistical analyses were performed using SPSS (IBM SPSS), and GraphPad Prism using Student t-tests for assessment of changes in the thickness of epidural tumor using a modified ESCC classification. A p value $\leq 0.05$ was considered statistically significant.

\section{Results}

A total of 8 patients ( 3 males and 5 females) were treated with thermal ablation and percutaneous spinal stabilization. The median age was 57 years (range 36-80 years). The primary histology was melanoma $(\mathrm{n}=3)$, lung $(\mathrm{n}=$ $3)$, thyroid $(n=1)$, and renal cell carcinoma $(n=1)$. Seven procedures were performed in the thoracic spine and 1 in the lumbar spine. All cases were performed under general anesthesia with the patient in the prone position. No morbidity was associated with the placement of the laser catheters, thermal ablation, or percutaneous instrumentation. The median preoperative Karnofsky Performance Scale (KPS) score was 60 (range 40-80). The median SINS score was 13 (range 12-16) (Table 1). To quantify 
TABLE 1. Characteristics in patients who underwent sLITT

\begin{tabular}{|c|c|c|c|c|c|c|c|c|c|c|}
\hline $\begin{array}{l}\text { Case } \\
\text { No. }\end{array}$ & $\begin{array}{l}\text { Age (yrs) } \\
\text { Sex }\end{array}$ & $\begin{array}{l}\text { Primary } \\
\text { Tumor }\end{array}$ & Level & $\begin{array}{l}\text { Preop KPS } \\
\text { Score }\end{array}$ & $\begin{array}{l}\text { SINS } \\
\text { Score }\end{array}$ & $\begin{array}{l}\text { Hospital } \\
\text { Stay (days) }\end{array}$ & $\begin{array}{l}\text { Treatment } \\
\text { Type }\end{array}$ & $\begin{array}{c}\text { Time Btwn Treatment } \\
\text { \& RT (days) }\end{array}$ & $\begin{array}{l}\text { Radiation Dose } \\
\text { (Gy/fraction) }\end{array}$ & Remark \\
\hline 1 & $46, \mathrm{M}$ & RCC & $\mathrm{T}-12$ & 70 & 13 & 18 & SRS & 3 & $24 / 1$ & Nephrectomy \\
\hline 2 & $80, M$ & Melanoma & T7-9 & 50 & 13 & 3 & NA & NA & NA & Prior cEBRT \\
\hline 3 & $56, \mathrm{M}$ & Lung & $\mathrm{T}-2$ & 60 & 14 & 5 & cEBRT & 4 & $30 / 10$ & \\
\hline 4 & $81, F$ & Lung & $L-1$ & 40 & 13 & 5 & SRS & 10 & $27 / 3$ & Prior cEBRT \\
\hline 5 & $63, F$ & Lung & T4-6 & 40 & 16 & 10 & NA & NA & NA & Prior cEBRT \\
\hline 6 & $60, F$ & Thyroid & $\mathrm{T}-11$ & 40 & 13 & 4 & NA & NA & NA & Prior cEBRT, SRS \\
\hline 7 & $37, \mathrm{~F}$ & Melanoma & T3-4 & 70 & 14 & 3 & NA & NA & NA & Prior SRS \\
\hline 8 & $36, F$ & Melanoma & $\mathrm{T}-10$ & 80 & 12 & 5 & cEBRT & 5 & $30 / 10$ & \\
\hline
\end{tabular}

$\mathrm{NA}=$ not available; $\mathrm{RCC}=$ renal cell carcinoma; $\mathrm{RT}$ = radiation therapy.

the improvement based on the ESCC score, we converted each category to a number as follows: $0=0 ; 1 \mathrm{a}=1 ; 1 \mathrm{~b}=2$; $1 \mathrm{c}=3 ; 2=4 ; 3=5$. The mean preoperative score was $4.5 \pm$ 0.27 ( \pm SD), which was significantly higher than the mean score of $2.75 \pm 0.37$ at a median 2.1 months (range 1.2-8.1 months) posttreatment ( $\mathrm{p}=0.0044$, Mann-Whitney test). Two patients were lost to follow-up because they were sent to hospice care after being discharged. For purpose of analysis, we considered their postoperative score to be similar to the preoperative score. The median number of laser fibers used to perform the treatment of the epidural tumor was 3 (range 2-6). Five patients had percutaneous stabilization inserted 1 segment above and below the tumor, and 3 patients had a construct that spanned 2 levels above and 2 below the lesion (Table 2). The median hospital stay was 5 days (range 3-18 days). One patient had an 18-day stay, which included the time to recover from the sLITT (6 days) and a nephrectomy to treat his primary renal cell carcinoma (12 days including inpatient rehabilitation). The median time to return to the oncological treatment (radiation after sLITT or chemotherapy) was 5 days (range 3-10 days).

\section{Discussion}

External-beam radiotherapy has been widely used as an effective treatment modality for spinal metastasis..$^{11,14}$ Unfortunately, it is well known that a subset of histologies present intrinsic resistance to cEBRT, ${ }^{8}$ and in such cases, patients treated with this modality alone may not achieve local control or adequate symptom palliation. ${ }^{17,18} \mathrm{~A}$ randomized trial $^{16}$ demonstrated that surgery followed by cEBRT is superior to radiotherapy alone in maintaining/ regaining sphincter function and capacity to walk in cases of epidural spinal cord compression. In addition to the functional benefit, surgery provided reduction in the need for corticosteroids and opioid analgesics when compared with cEBRT alone. However, this study did not provide information about local control, surgical morbidity, or time to resume oncological treatment for the primary tumor. Prognostic scores have been developed to predict survival and guide surgeons to tailor the extent of tumor resection to maximize surgical results..$^{24,25}$ This philosophy has shifted and evolved, aiming to optimize the utilization of surgery, to achieve the best balance between local control and adequate palliation and to avoid excessive morbid- ity, especially in patients with poor prognosis. ${ }^{23}$ Recent advances in radiobiology and image guidance led to the development of spinal SRS, which allows the delivery of a high dose of radiation in a single or hypofractionated fashion, with the hallmark of a rapid fall off outside a selected target. ${ }^{19,26}$ Several reports have demonstrated good local control in cases of unfavorable histologies to cEBRT and cemented the role of SRS in the management of spinal metastasis. $^{6-8,20,27}$ Nevertheless, in the setting of epidural disease leading to spinal cord displacement, the radiation dose prescribed to the tumor needs to be adjusted to respect the constraints of the spinal cord, ${ }^{19,21}$ which can be associated with treatment failure. ${ }^{3}$

Bilsky at al. ${ }^{1}$ proposed an algorithm in which neurological, oncological, mechanical, and systemic factors (NOMS) are analyzed and the utilization of cEBRT, SRS, and/or surgery is recommended based on tumor sensitivity to radiation and the extent of epidural involvement. In this context, local control is achieved with radiation therapy (either cEBRT or SRS), and surgery is used to remove the epidural tumor and stabilize the spine (separation surgery). The extent of resection loses importance in terms of local control, provided an adequate coverage with tumoricidal doses of stereotactic radiosurgery is delivered to the residual disease. ${ }^{13}$ Laufer et al. ${ }^{12}$ report a cumulative incidence of local progression of $4.1 \%$ and $9 \%$, respectively, using hypofractionated (24-30 Gy in 3 fractions) or high single-dose (24 Gy) SRS after separation surgery. Despite the concept of minimizing surgical morbidity and a faster

TABLE 2. Detail of treatment parameters

\begin{tabular}{|c|c|c|c|c|c|}
\hline \multirow{2}{*}{$\begin{array}{l}\text { Case } \\
\text { No. }\end{array}$} & \multicolumn{2}{|c|}{ ESCC Score } & \multirow{2}{*}{$\begin{array}{l}\text { No. of } \\
\text { Fibers }\end{array}$} & \multirow{2}{*}{$\begin{array}{c}\text { Level of } \\
\text { Instrumentation }\end{array}$} & \multirow{2}{*}{$\begin{array}{l}\text { Length of Imaging } \\
\text { Follow-Up (mos) }\end{array}$} \\
\hline & Preop & Postop & & & \\
\hline 1 & 3 & $1 \mathrm{~b}$ & 6 & T11-L1 & 8.1 \\
\hline 2 & 3 & $1 \mathrm{c}$ & 6 & T6-10 & 2.2 \\
\hline 3 & 3 & $1 b$ & 2 & T1-3 & 4.8 \\
\hline 4 & 2 & 2 & 3 & T12-L2 & 2.1 \\
\hline 5 & 2 & NA & 4 & T2-3 to T7-8 & NA \\
\hline 6 & 3 & $1 a$ & 3 & T10-L2 & 1.6 \\
\hline 7 & 3 & $1 \mathrm{c}$ & 4 & T5-7 & 1.2 \\
\hline 8 & 2 & NA & 2 & T10-12 & NA \\
\hline Median & 3 & $1 \mathrm{c}$ & 3 & & 2.1 \\
\hline
\end{tabular}


recovery, there have been no data confirming an advantage of separation surgery over a more extensive intralesional resection in terms of lower surgical morbidity and faster return to systemic treatment.

We present the first case series combining percutaneous spinal stabilization with laser interstitial thermotherapy for treatment of high-grade malignant spinal cord compression. Our philosophy is based on the concept of separation surgery put forth by Bilsky and Smith, ${ }^{1}$ in which the epidural disease is ablated prior to the SRS treatment. However, as demonstrated in this series, we applied this method as salvage therapy in 3 patients who underwent cEBRT, without improvement in the epidural compression, and in 2 patients in whom prior spinal SRS had failed. Since surgical morbidity with sLITT is low, we believe that patients with poor performance experience a benefit with respect to a shorter hospital stay and return to oncological treatment in less than a week.

All patients in this series underwent placement of posterior segmental instrumentation via the percutaneous technique. When compared with our prior experience, this increased the median length of hospital stay from 2 to 5 days. The most common reason for hospital stay was the time to achieve good pain control. Although we cannot distinguish the amount of pain related to ablation and with the trauma in the soft tissue created by the insertion of pedicle screws, we believe the latter constitutes the most likely reason. Despite this, we believe that our cohort had a shorter hospitalization than if the procedure had been performed in the standard open technique.

We have improved the surgical workflow by performing MRI of the patient in the operative position and using these images as the platform for image guidance for laser catheter placement. We have studied the implantation of 47 laser probes and found a median error of $0.7 \mathrm{~mm}$ (range 0-3.2 $\mathrm{mm}$ ) in the accuracy of the image-guided system (unpublished data). We believe that performing placement of percutaneous screws inside the iMRI room is safe and does not significantly increase the operative time. In this case, navigation can be reregistered based on the ink marks of the previously scanned fiducials. We still recommend verification of accuracy with fluoroscopic images using the pedicle as a landmark prior to advancing the Jamshidi needles for placement of posterior segmental instrumentation.

An evaluation of the effectiveness of laser ablation in controlling the epidural tumor is beyond the scope of this report and will be the focus of future publications. In the short follow-up of this series, we have demonstrated good clinical local control in 6 of 8 patients, where the median ESCC score decreased 2 points from 3 to $1 \mathrm{c}$. Of note, the median KPS score of this cohort was 60 , which indicates a relatively compromised population, in whom an extensive operation may be associated with very high morbidity.

\section{Conclusions}

This report demonstrates our initial experience with the concept of percutaneous separation surgery. Among the several limitations of this study, the retrospective nature, the small size of the population, the short follow-up, the heterogeneity of histologies, and the lack of comparison with the standard surgical technique may limit the interpretation of our results. A randomized prospective study comparing open and percutaneous separation surgery will be needed to evaluate the role of this new concept in the management of ESCC. Nevertheless, we believe it can be used as a less invasive alternative to provide palliation for patients who are poor surgical candidates and cannot afford long interruptions in systemic treatment.

\section{References}

1. Bilsky M, Smith M: Surgical approach to epidural spinal cord compression. Hematol Oncol Clin North Am 20:13071317,2006

2. Bilsky MH, Laufer I, Fourney DR, Groff M, Schmidt MH, Varga PP, et al: Reliability analysis of the epidural spinal cord compression scale. J Neurosurg Spine 13:324-328, 2010

3. Chang EL, Shiu AS, Mendel E, Mathews LA, Mahajan A, Allen PK, et al: Phase I/II study of stereotactic body radiotherapy for spinal metastasis and its pattern of failure. J Neurosurg Spine 7:151-160, 2007

4. Fisher CG, DiPaola CP, Ryken TC, Bilsky MH, Shaffrey CI, Berven SH, et al: A novel classification system for spinal instability in neoplastic disease: an evidence-based approach and expert consensus from the Spine Oncology Study Group. Spine (Phila Pa 1976) 35:E1221-E1229, 2010

5. Frankel HL, Hancock DO, Hyslop G, Melzak J, Michaelis LS, Ungar GH, et al: The value of postural reduction in the initial management of closed injuries of the spine with paraplegia and tetraplegia. I. Paraplegia 7:179-192, 1969

6. Gerszten PC, Burton SA, Ozhasoglu C, Vogel WJ, Welch WC, Baar J, et al: Stereotactic radiosurgery for spinal metastases from renal cell carcinoma. J Neurosurg Spine 3:288295, 2005

7. Gerszten PC, Burton SA, Ozhasoglu C, Welch WC: Radiosurgery for spinal metastases: clinical experience in 500 cases from a single institution. Spine (Phila Pa 1976) 32:193-199, 2007

8. Gerszten PC, Mendel E, Yamada Y: Radiotherapy and radiosurgery for metastatic spine disease: what are the options, indications, and outcomes? Spine (Phila Pa 1976) 34 (22 Suppl):S78-S92, 2009

9. Ghia AJ, Rebueno NC, Li J, Brown PD, Rhines LD, Tatsui $\mathrm{CE}$ : The use of image guided laser interstitial thermotherapy to supplement spine stereotactic radiosurgery to manage metastatic epidural spinal cord compression: Proof of concept and dosimetric analysis. Pract Radiat Oncol 6:e35-e38, 2016

10. Harris EB, Massey P, Lawrence J, Rihn J, Vaccaro A, Anderson DG: Percutaneous techniques for minimally invasive posterior lumbar fusion. Neurosurg Focus 25(2):E12, 2008

11. Latini P, Maranzano E, Ricci S, Aristei C, Checcaglini F, Panizza BM, et al: Role of radiotherapy in metastatic spinal cord compression: preliminary results from a prospective trial. Radiother Oncol 15:227-233, 1989

12. Laufer I, Iorgulescu JB, Chapman T, Lis E, Shi W, Zhang Z, et al: Local disease control for spinal metastases following "separation surgery" and adjuvant hypofractionated or highdose single-fraction stereotactic radiosurgery: outcome analysis in 186 patients. J Neurosurg Spine 18:207-214, 2013

13. Laufer I, Rubin DG, Lis E, Cox BW, Stubblefield MD, Yamada Y, et al: The NOMS framework: approach to the treatment of spinal metastatic tumors. Oncologist 18:744-751, 2013

14. Maranzano E, Latini P: Effectiveness of radiation therapy without surgery in metastatic spinal cord compression: final results from a prospective trial. Int J Radiat Oncol Biol Phys 32:959-967, 1995 
15. Moussazadeh N, Rubin DG, McLaughlin L, Lis E, Bilsky $\mathrm{MH}$, Laufer I: Short-segment percutaneous pedicle screw fixation with cement augmentation for tumor-induced spinal instability. Spine J 15:1609-1617, 2015

16. Patchell RA, Tibbs PA, Regine WF, Payne R, Saris S, Kryscio RJ, et al: Direct decompressive surgical resection in the treatment of spinal cord compression caused by metastatic cancer: a randomised trial. Lancet 366:643-648, 2005

17. Rades D, Karstens JH, Alberti W: Role of radiotherapy in the treatment of motor dysfunction due to metastatic spinal cord compression: comparison of three different fractionation schedules. Int J Radiat Oncol Biol Phys 54:1160-1164, 2002

18. Rades D, Stalpers LJ, Hulshof MC, Borgmann K, Karstens $\mathrm{JH}$, Koning CC, et al: Comparison of 1 x 8 Gy and 10 x 3 Gy for functional outcome in patients with metastatic spinal cord compression. Int J Radiat Oncol Biol Phys 62:514-518, 2005

19. Ryu S, Yoon H, Stessin A, Gutman F, Rosiello A, Davis R: Contemporary treatment with radiosurgery for spine metastasis and spinal cord compression in 2015. Radiat Oncol J 33:1-11, 2015

20. Sahgal A, Larson DA, Chang EL: Stereotactic body radiosurgery for spinal metastases: a critical review. Int J Radiat Oncol Biol Phys 71:652-665, 2008

21. Sahgal A, Weinberg V, Ma L, Chang E, Chao S, Muacevic A, et al: Probabilities of radiation myelopathy specific to stereotactic body radiation therapy to guide safe practice. Int J Radiat Oncol Biol Phys 85:341-347, 2013

22. Tatsui CE, Stafford RJ, Li J, Sellin JN, Amini B, Rao G, et al: Utilization of laser interstitial thermotherapy guided by real-time thermal MRI as an alternative to separation surgery in the management of spinal metastasis. J Neurosurg Spine 23:400-411, 2015

23. Tokuhashi Y, Ajiro Y, Umezawa N: Outcome of treatment for spinal metastases using scoring system for preoperative evaluation of prognosis. Spine (Phila Pa 1976) 34:69-73, 2009
24. Tokuhashi Y, Matsuzaki H, Oda H, Oshima M, Ryu J: A revised scoring system for preoperative evaluation of metastatic spine tumor prognosis. Spine (Phila Pa 1976) 30:2186-2191, 2005

25. Tomita K, Kawahara N, Kobayashi T, Yoshida A, Murakami H, Akamaru T: Surgical strategy for spinal metastases. Spine (Phila Pa 1976) 26:298-306, 2001

26. Wang XS, Rhines LD, Shiu AS, Yang JN, Selek U, Gning I, et al: Stereotactic body radiation therapy for management of spinal metastases in patients without spinal cord compression: a phase 1-2 trial. Lancet Oncol 13:395-402, 2012

27. Yamada Y, Bilsky MH, Lovelock DM, Venkatraman ES, Toner S, Johnson J, et al: High-dose, single-fraction imageguided intensity-modulated radiotherapy for metastatic spinal lesions. Int J Radiat Oncol Biol Phys 71:484-490, 2008

\section{Disclosures}

The authors report the following: Dr. Rhines, consultant for Stryker and Globus; Dr. Li, support of non-study-related clinical or research effort from Medtronic.

\section{Author Contributions}

Conception and design: Tatsui, Li, Ghia. Acquisition of data: Tatsui. Analysis and interpretation of data: Tatsui, Belsuzarri, Oro, Amini, Espinoza, Rao. Drafting the article: Tatsui, Belsuzarri, Oro, Rao. Critically revising the article: Tatsui, Rhines, Li, Ghia, Amini, Brown, Rao. Reviewed submitted version of manuscript: Tatsui, Rhines, Rao. Approved the final version of the manuscript on behalf of all authors: Tatsui.

\section{Correspondence}

Claudio E. Tatsui, Department of Neurosurgery, University of Texas, MD Anderson Cancer Center, 1515 Holcombe Blvd., Unit 442, Houston, TX 77030. email:cetatsui@mdanderson.org. 